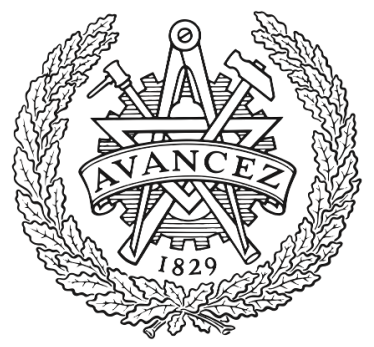

CHALMERS

UNIVERSITY OF TECHNOLOGY

\title{
Towards Modelling and Design of Magnetostrictive Electric Generators
}

Downloaded from: https://research.chalmers.se, 2023-04-26 15:13 UTC

Citation for the original published paper (version of record):

Berbyuk, V., Sodhani, J. (2005). Towards Modelling and Design of Magnetostrictive Electric Generators. Proc. of II ECCOMAS Thematic Conference on Smart Structures and Material, Lisbon, July 18-21, 2005, Eds. C. A. Mota Soares et al., Lisbon, 2005,: 1-16

N.B. When citing this work, cite the original published paper. 


\section{ECCOMAS Thematic Conference}

on Smart Structures and Materials

\section{Paper}

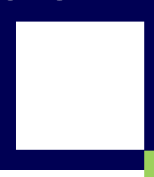

\section{Instifuło Superior Técnico, Lisbon 18 / 21 July 2005}




\title{
TOWARDS MODELLING AND DESIGN OF MAGNETOSTRICTIVE ELECTRIC GENERATORS
}

\author{
Viktor Berbyuk and Jayesh Sodhani
}

\author{
Mechanical Systems Division \\ Department of Applied Mechanics \\ Chalmers University of Technology \\ SE-412 96, Göteborg, Sweden \\ e-mail: viktor.berbyuk@chalmers.se,e-mail: jayesh.sodhani@chalmers.se \\ web page: http://www.chalmers.se
}

Keywords: Smart Materials, Magnetostriction, Magnetomechanical Effect, Power Harvesting from Vibration, Villari Effect, Magnetostrictive Electric Generator, Terfenol-D.

\begin{abstract}
During the last decades the interest in research and development of smart actuators, sensors and power generators that used giant magnetostrictive materials is continually growing. Both academia and industry are actively looking for bread utilization of this technology for different applications (active vibration and noise control, structural health monitoring, self-powered electronic equipments and systems, MEMS, robotics, biomedical engineering, etc.). The proposed paper is in the field of applications of novel highly magnetostrictive materials for power harvesting, namely vibration-to-electric energy conversion. The term "power harvesting" is used for process of acquiring the energy surrounding a system and converting it into usable electrical energy. The problem of modelling and design of magnetostrictive electric generators (MEG) are considered. The fundamental basic for design of MEG is a Villari effect. That is, by applying a mechanical stress to a magnetostrictive material, the magnetization along the direction of the applied stress of the material varies due to the magnetostrictive effect. The flux variation obtained in the material induces an emf in a coil surrounding the material. The brief review on research and development of power generators using smart materials is given. Original MEG and the respective test rig which were built for study fundamentals of transduction processes of mechanical energy of vibrating structures into electrical energy are presented. Terfenol -D rod with $50 \mathrm{~mm}$ in length and $15 \mathrm{~mm}$ in diameter is used as an active material in MEG design. Test rig's measurement data have confirmed the expected performance of the MEG. These data are used for validation of the mathematical model of MEG that was developed and implemented in Matlab/Simulink environment.
\end{abstract}




\section{INTRODUCTION}

The study of magnetostriction began in 1842 when James P. Joule first observed that a sample of iron changes its length when magnetized with a magnetic field. The term magnetostriction refers to any change in dimension of a magnetic material caused by a change in its magnetization. The Joule magnetostriction is commonly employed in actuator and sensor applications [1-3]. Because magnetostriction is an inherent property of magnetic materials, it does not degrade over time as can be the case with some piezoelectric substances. Furthermore, newer magnetostrictive materials provide strains, forces, energy densities, and coupling coefficients which compete advantageously with more established transducer technologies such as those based on piezoelectric materials.

One of the first studied applications of highly magnetostrictive materials was as a generator of force and motion for underwater sound sources. Started in the 1960s, the exceptional force and strain capabilities of these materials motivated the U. S. Navy to carry out extensive research in this field [1]. Many potential applications of highly magnetostrictive materials were then suggested, for instance sound and vibration sources, sonar systems, mechanical impact actuators, active vibration control and structural health monitoring, [1-3].

Recent developments in miniaturized sensors, digital processors, self-powered electronics and wireless communication systems have many desirable applications. The realization of these applications however, is limited by the lack of a similarly sized power sources. Powering the above mentioned systems can be a significant engineering problem, as traditional solutions such as batteries are not always appropriate. The one issue that still needs to be resolved is a method to generate sufficient energy to power the electronics.

To day micro-scale concepts to generate electrical power include devices which use the stored energy in fuels to those which harvest energy from environment. The term "power harvesting" is used for process of acquiring the energy surrounding a system and converting it into usable electrical energy. The energy conversion of light, thermal or mechanical sources is an important aspect for power generations. In the last few years, there has been a surge of research in the area of power harvesting from vibration using piezoelectric materials [4-19]. Of primary importance is the efficiency of power conversion. Many proposed power harvesting systems employ a piezoelectric component to convert the mechanical energy to electrical energy. The major limitation facing researchers in the field of power harvesting revolve around the fact that the power generated by piezoelectric materials is far too small to power most electronics. While piezoelectric materials are now major method of harvesting energy [4], other methods do exist; for example, methods based on Villari effect of magnetostrictive materials $[1,12,24]$. Novel magnetostrictive materials are probably most prospective materials for vibration-to-electric energy conversion since they have high energy density and very high electromechanical coupling effect. Methods of increasing the amount of energy generated by power harvesting device or developing new and innovative methods of accumulating the energy are the key technologies that will allow power harvesting to become a source of power for portable electronics, wireless sensors and other microelectromechanical systems [4]. Operation of power harvesting devices and other transducers that used magnetostrictive materials are based on dynamic interaction between magnetic and electric 
fields, inherent elastic properties of active material and mechanical external loads. To be able to analyze the energy transduction processes, design and optimize the performance of magnetostrictive power harvesting devices and other magnetostrictive transducers, they must be considered as complex magnetoelastic electromechanical systems.

Due to above mentioned there is a lot of ongoing research activity all over the world in the field of smart structures and smart material based transducers technology both in academia and industry. Results of the research and development that are dealing with giant magnetostrictive materials and are most close related to the topic of the paper can be found, for instance in [ 1, 3, 5, 14-17, 19-30].

The proposed paper is in the field of application of novel highly magnetostrictive materials for power harvesting, namely in the field of vibration-to-electric energy conversion. The approach for modeling of performance of MEG as a power harvesting device is described. The novel MEG was built and the experimental results of the generator performance are presented.

\section{MATHEMATICAL MODELING OF MAGNETOSTRICTIVE ELECTRIC GENERATOR}

A characteristic property of magnetostrictive materials (active material in magnetostrictive transducers) is that a mechanical strain will occur if they are subjected to a magnetic field in addition to strain originated from pure applied stresses. Also, their magnetization changes due to changes in applied mechanical stresses in addition to the changes caused by changes of the applied magnetic field. These dependencies can be described by mathematical functions [1]:

$$
\begin{aligned}
& \varepsilon=\varepsilon(\sigma, H) \\
& B=B(\sigma, H)
\end{aligned}
$$

Here $\varepsilon, \sigma, H$ be the strain, the stress and the applied magnetic field strength, respectively, $B$ be the magnetic flux density.

Taking into account all above mentioned and the fact that the most important mode of operation of magnetostrictive materials is the longitudinal one, the linearization of the differential response of strain and magnetization (relations (2) and (3)) leads to the following equations of magnetomechanical coupling:

$$
\begin{aligned}
& \varepsilon=S^{H} \sigma+d_{33} H \\
& B=d_{33}^{*} \sigma+\mu^{\sigma} H
\end{aligned}
$$


Here:

$S^{H}=\frac{\partial \varepsilon}{\partial \sigma}{ }_{\mid H=\text { const }}=\frac{1}{E^{H}}$, where $E^{H}$ be the Young's modulus at constant applied magnetic field strength,

$d_{33}=\frac{\partial \varepsilon}{\partial H}{ }_{\mid \sigma=c o n s t}$ be the magnetostrictive strain derivative (linear coupling coefficient),

$d_{33}^{*}=\frac{\partial B}{\partial \sigma}{ }_{\mid H=\text { const }}$ be the magnetomechanical effect,

$\mu^{\sigma}=\frac{\partial B}{\partial H}{ }_{\mid \sigma=c o n s t}$ be the magnetic permeability at a constant stress.

Assume that a MEG is integrated into a moving structure (hosting system), the phase state of which is determined by the phase vector $\{\mathbf{y}(t), \dot{\mathbf{y}}(t)\}$. The interaction between the dynamics of hosting system and the dynamics of a MEG can be expressed by the following relation:

$$
\sigma(\mathbf{y}, \dot{\mathbf{y}})=f(\mathbf{y}, \dot{\mathbf{y}}, \mathbf{c})
$$

Here $\sigma(\mathbf{y}, \dot{\mathbf{y}})$ be the stress applied to active material of MEG, the function $f(\mathbf{y}, \dot{\mathbf{y}}, \mathbf{c})$ and the vector parameter $\mathbf{c}$ are determined by the given magnetic design of a magnetostrictive transducer and by the design of adaptive structure that needed to integrate the transducer into hosting system.

It's not necessary to assume the coefficients $E^{H}, \mu^{\sigma}, d_{33}, d_{33}^{*}$ are single valued or linear, however this is generally approach taken in many investigations of performance of magnetostrictive transducers [21].

Several important assumptions are built into the magnetostrictive model (equations (4), (5)). First, linear operation of active material (Terfenol-D, [1]) in the magnetostrictive transducer is assumed. Although the magnetostrictive effect is nonlinear, for low signal levels, less than approximately one-third the maximum strain capability, the linear equations of magnetostriction provide a good first approximation [21]. Second, the magnetostriction process is assumed to be reversible according to

$$
d_{33}=\frac{\partial \varepsilon}{\partial H_{\mid \sigma=c o n s t}}=\frac{\partial B}{\partial \sigma}{ }_{\mid H=\text { const }}=d_{33}^{*}
$$


Finally, the strain, the stress, the magnetic field, and the magnetic flux are assumed to be uniform throughout the active material.

Equations (1) - (7) constitute the mathematical model of a magnetoelastic mechanical system modeled magnetostrictive transducer integrated into a hosting system. As indicated in (4)-(6), it is assumed that the stress $\sigma$ depends on phase state of the hosting system $(\mathbf{y}, \dot{\mathbf{y}})$, i.e. the dynamics of the hosting system affects the performance of the magnetostrictive transducer.

Consider the following problem.

Problem A. Assume that due to interaction of a MEG with the hosting system the following stress is acting on the active material of the magnetostrictive transducer: $\sigma=f\left(\mathbf{y}_{a}(t), \dot{\mathbf{y}}_{a}(t), \mathbf{c}\right)$. It's required to determine the voltage and the electrical power of a MEG.

Solution of the Problem A can be obtained by the following procedure.

From equations (5) and (10) it can be written

$$
B=d_{33}^{*} f\left(\mathbf{y}_{a}(t), \dot{\mathbf{y}}_{a}(t), \mathbf{c}\right)+\mu^{\sigma} H
$$

The action of the applied stress produces a changing flux density $B$ which according to Faraday's law induces a voltage, $U(t)$, in a coil surrounding the Terfenol-D sample such that:

$$
U(t)=-N A \frac{d B}{d t}
$$

where $N$ and $A$ are the number of turns and the cross section area of coil.

This voltage will generate a current, $I$, in the coil, that according to Lenz's law will give rise to an opposing field, $H_{r}$ :

$$
H_{r}=\frac{N I}{l}
$$

where $l$ is the length of coil.

Using the equations (8), (9) and Ohm's law

$$
I=U / R
$$

the total applied field, $H$, will be given by formula 


$$
H=H_{0}+H_{r}=H_{0}-\frac{N^{2} A}{R l} \frac{d B}{d t}
$$

where $H_{0}$ is the steady magnetic field, $R=R_{0}+r, R_{0}$ is the electric circuit load, and $r$ is the coil resistance.

Substituting expression (11) into equation (7) the following differential equation for magnetic flux will be obtained:

$$
\frac{d B(t)}{d t}+c B(t)=f_{a}(t)
$$

Here

$$
\begin{aligned}
& c=R l /\left(\mu^{\sigma} N^{2} A\right) \\
& f_{a}(t)=c\left(\mu^{\sigma} H_{0}+d_{33}^{*} f\left(\mathbf{y}_{a}(t), \dot{\mathbf{y}}_{a}(t), \mathbf{c}\right)\right)
\end{aligned}
$$

The solution to equation (12) can be found and be of the form

$$
B(t)=e^{-c t}\left(B_{0}+\int_{0}^{t} f_{a}(\tau) e^{c \tau} d \tau\right)
$$

where $B_{0}=B(0)$ is initial magnetic flux.

Using (8), (10), (12)-(14), and (3) the induced voltage, $U(t)$, and strain, $\varepsilon(t)$, in the magnetostrictive transducer are determined by the following expressions:

$$
\begin{gathered}
U(t)=-N A f_{a}(t)+c N A e^{-c t}\left[B_{0}+\int_{0}^{t} f_{a}(\tau) e^{c \tau} d \tau\right] \\
\varepsilon(t)=\frac{1}{E^{H}} f\left(\mathbf{y}_{a}(t), \dot{\mathbf{y}}_{a}(t), \mathbf{c}\right)+d_{33}\left(H_{0}-\frac{1}{c \mu^{\sigma}}\left(f_{a}(t)-c B(t)\right)\right.
\end{gathered}
$$

Here $c, \quad f_{a}(t), \quad B(t)$ are determined by formulae (13) and (14), respectively.

Having the induced voltage (15), the electrical power of MEG can be evaluated as follows

$$
P(t)=U^{2}(t) / R
$$


As an example of utilization of the developed algorithm, the time histories of voltages of a MEG for different frequencies of external excitations are plotted in Fig. 1. These curves were calculated by using formula (15). The external stress acting on the Terfenol-D rod was given by the expression:

$$
\sigma=\sigma_{0}+\sigma_{a} \sin (w t)
$$

The values of the input parameters were chosen as follows:

$$
\begin{aligned}
& E^{H}=3.35 \times 10^{10} \mathrm{~Pa}, \quad \mu^{\sigma}=6, \quad d_{33}=d_{33}{ }^{*}=1.79 \times 10^{-8} \\
& H_{0}=50 \mathrm{kA} / \mathrm{m}, \quad \sigma_{0}=6.9 \mathrm{MPa}, \quad \sigma_{a}=100 \mathrm{MPa} \\
& R=1 \Omega, \quad N=116, \quad l=2.02 \mathrm{~m}, \quad A=1.54 \times 10^{-6} \mathrm{~m}
\end{aligned}
$$

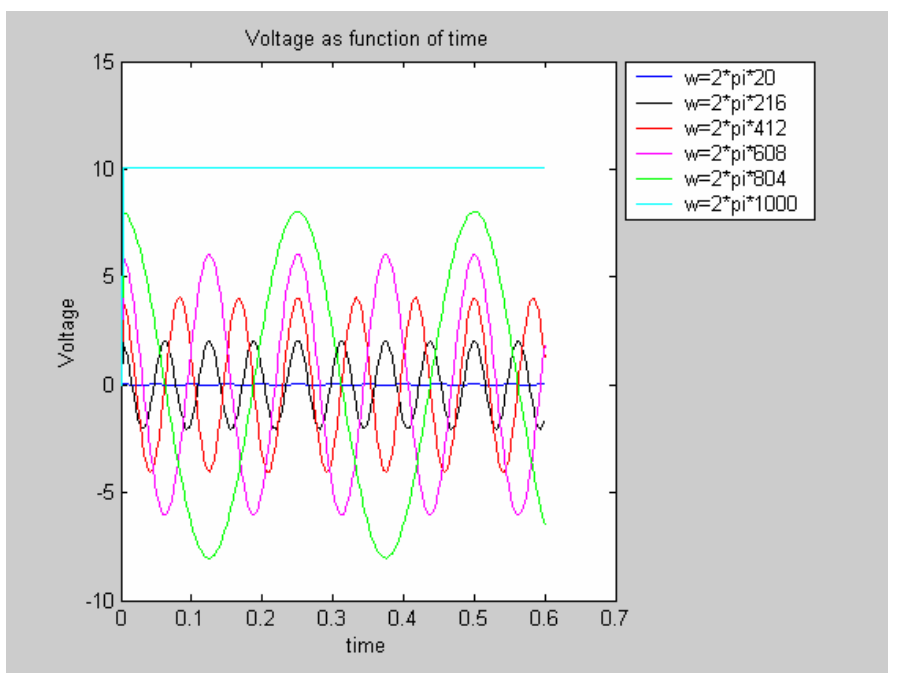

Figure 1. Voltage time histories of a MEG for different frequencies of external excitations.

The developed above algorithm was implemented in Matlab/Simulink with user friendly interface. The algorithm is based on linearized relations (4), (5) and can be used for many applications within the frame of above listed assumptions, for instance for prediction of the performance of magnetostrictive transducers, optimization of their magnetic design, etc.

\section{CHALMERS MAGNETOSTRICTIVE ELECTRIC GENERATOR}

The new MEG was manufactured, assembled and installed at Chalmers University of Technology. Terfenol-D rod with length $50 \mathrm{~mm}$ and $15 \mathrm{~mm}$ in diameter is used as core element in MEG. The rod is subjected to mechanical prestress input, hence compressing it and forcing 
the magnetic dipoles to turn. The magnetization of Terfenol-D by permanent magnets can be varied with different number of magnets that are filled into the magazine. Terfenol-D rod is fitted into a bobbin and a transformer coil is wound around it. The bobbin can be used as add on sub part to the assembly of the MEG. The change in magnetic field will result in induced electric current in the electrical coil.

The experimental setup comprises of the test rig, the MEG, Oscilloscope (HP, 4 channel $100 \mathrm{MHz}$ ), and Amplifier, Frequency / speed controller of the motor, (Fig. 2).

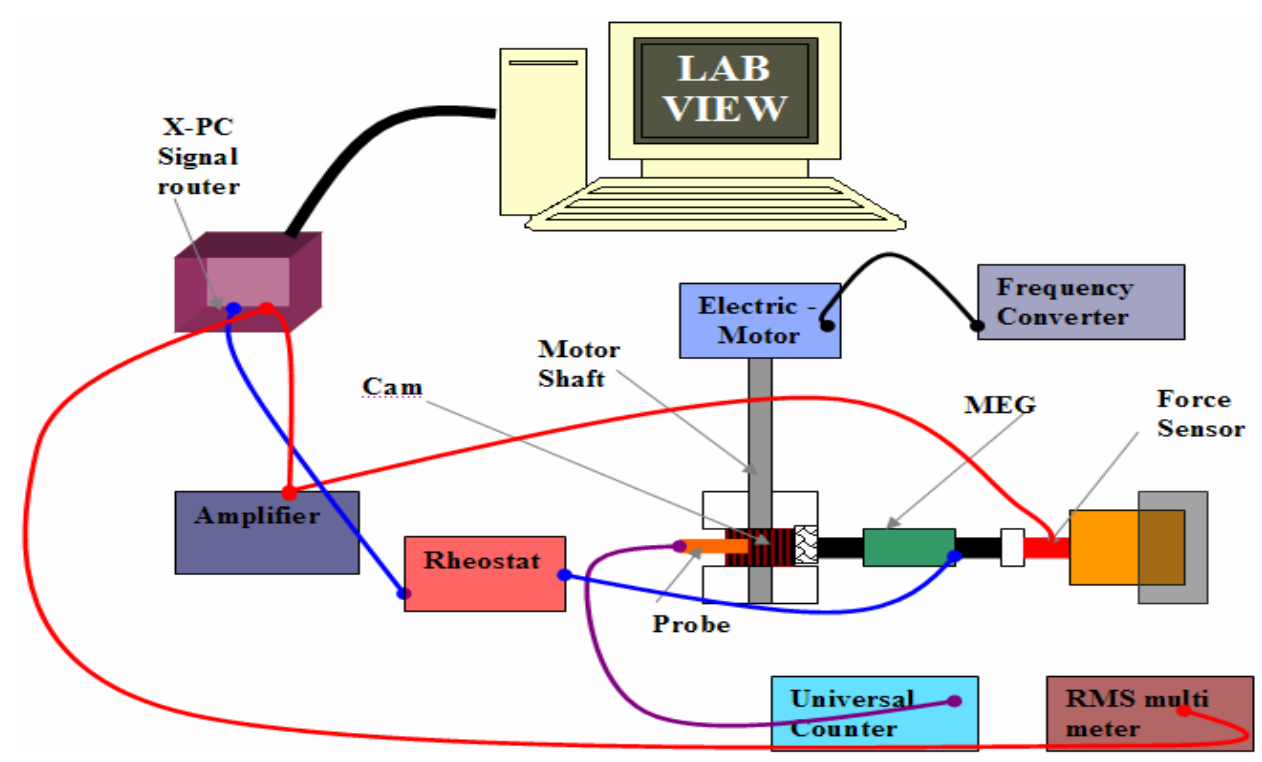

Figure 2. Experimental measurement set up for MEG

A high frequency test rig is used for vibration excitation of the Terfenol-D by a cam coupled to electric motor shaft. The facet on the cam profile is machined to give a sinus wave input to the MEG. The force sensor (4 strain gauges) is used to measure the mechanical force input to the MEG. The wiring arrangement is a Wheatstone bridge that is fixed to an adaptor and connected to the amplifier (Bridge amplifier). The data of the force and voltage as output can be obtained on the oscilloscope. Root mean square (RMS) multimeter is used to govern the mechanical prestress on the Terfenol-D. There is small DC signal that is generated in the strain gauge due to the sinus excitation by the cam to the plunger transmitting the vibration to the MEG. This signal is amplified in the amplifier and tuned for 1 volt $=1000 \mathrm{~N}$ scale after amplification, i.e. for every one volt measured on the oscilloscope when mechanical prestress is applied to the moving carriage $1000 \mathrm{~N}$ is the force that is obtained in the MEG. The speed of the motor and hence the frequency of external excitation to the MEG is controlled by an electrical frequency converter. The output of the MEG is an AC voltage. The AC voltage can also be rectified and regulated to some cut of value of some choice by a voltage regulator. 
There are two outputs signals that can be read on Lab View plots. The force sensor signal (red) is amplified for 1 volt $=1000 \mathrm{~N}$ on the amplifier (HBM ME10), (refer Fig.2). The second signal (blue) is the electric peak-to- peak voltage of MEG coil to rheostat where the resistance is set to $1 \Omega$. This signal is then directed to a bridge (X-PC signal router) between the computer measurement card and scan signals rates. Frequency of the test rig is read with a probe that constantly converts the cam speed, (rpm) to $\mathrm{Hz}$ (see orange probe in Fig. 2). Strain gauge is used to measure the mechanical force applied to the MEG. This signal can also be read on the RMS multimeter.

Two type of Terfenol-D rod are used in the experiments. One rod has been laminated from four Terfenol-D pieces. The reason for the lamination is to minimize the eddy current losses in the Terfenol-D rod due to current circulating it the material. Another rod is a non laminated Terfenol-D rod from ETREMA products, Inc., USA, (refer Fig 3.)

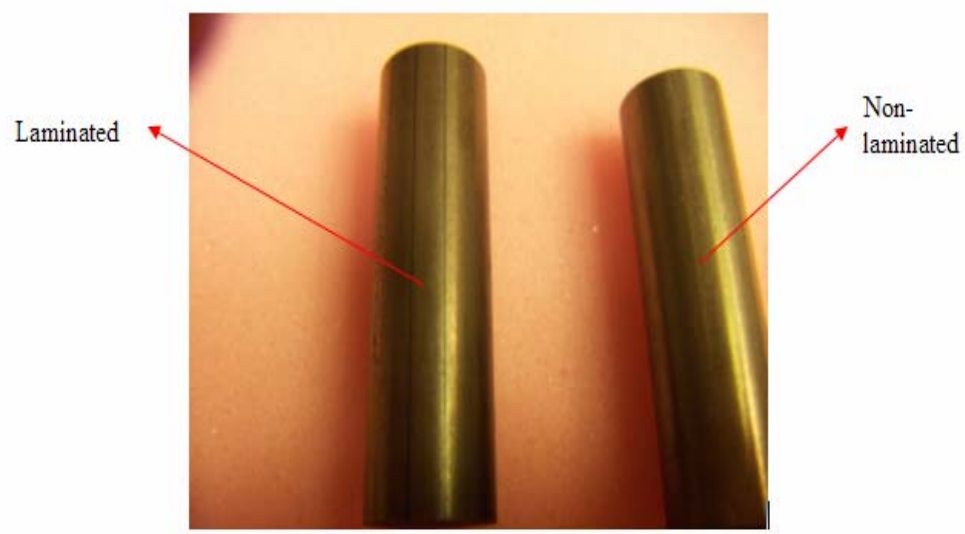

Figure 3. Terfenol-D rod samples

The input parameters of the test rig that can be varied are frequency and amplitude of external vibration, and mechanical prestress. Outputs are the voltage, the current and power.

\section{MEASUREMENTS}

The experiments are carried out for Terfenol-D rod of length $50 \mathrm{~mm}$ and diameter $15 \mathrm{~mm}$ for laminated sample. The permanent magnets TBD alloy used to create magnetic bias for the Terfenol-D rod. The magnets are round shaped in $6 \mathrm{~mm}$ diameter and $2.5 \mathrm{~mm}$ height. Due to design restrictions the number of magnets that can be used are 320 . The amplitude of excitation, $a$, was equal to $0.000135 \mathrm{~m}$. It was measured by using a dial gauge on the cam facet.

Mechanical power input (MPI) and electric power output (EPU) were calculated by using the following formulae: 


$$
\begin{aligned}
& M P I=F^{*} f^{*} a \\
& E P U=\frac{\left(\left(U_{p p}\right) / 2\right)^{2}}{R}
\end{aligned}
$$

Here, $F$ be the input external excitation force, in $\mathrm{N}, f$ be the frequency of excitation, in $\mathrm{Hz}, a$ be the amplitude of excitation, in $\mathrm{m}, U_{p p}$ be the peak-to-peak voltage, in $\mathrm{V}, R$ be the resistance, in $\Omega$.

Efficiency of the MEG (Power Harvesting, $P H, \%$ ) is estimated by the following expression :

$$
P H(\%)=\frac{E P U}{M P I} * 100
$$

\section{RESULTS}

The following graphs (Fig. 4 and Fig. 5) give a comparison between the MPI VS EPU and power harvesting efficiency of MEG for different mechanical prestresses, input external excitation forces and frequencies. Here the coil resistance is equal to $1 \Omega$, (refer, Table 1 for the data).

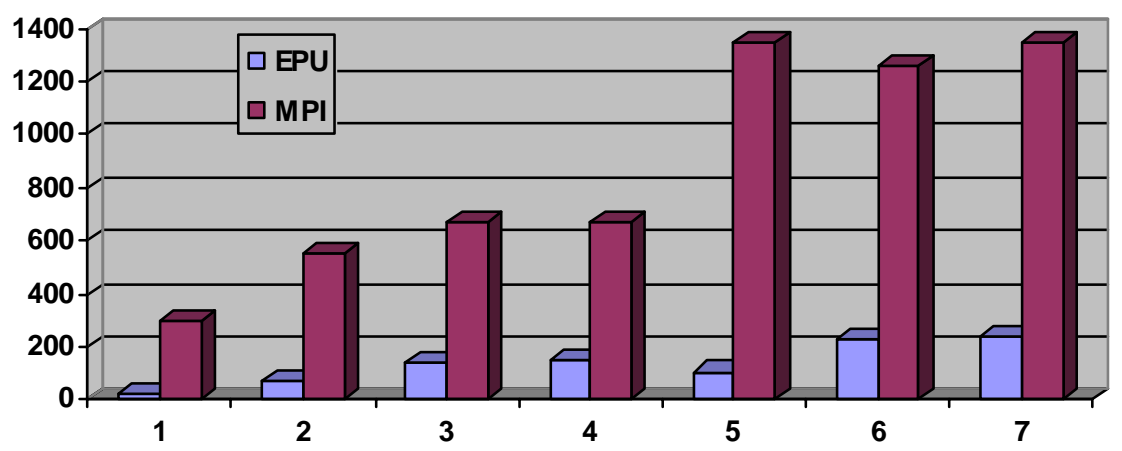

Figure 4. Bar chart comparison between MPI VS EPU

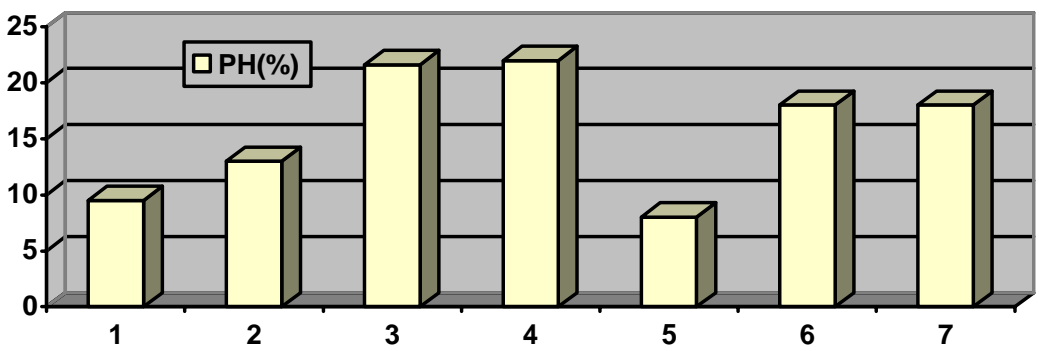

Figure 5. Bar chart for the PH (\%) for each case listed in Table 1 


\begin{tabular}{|c|c|c|c|c|c|c|c|}
\hline Bar Number & 1 & 2 & 3 & 4 & 5 & 6 & 7 \\
\hline Mechanical Prestress, N & 1000 & 2000 & 2500 & 2700 & 2000 & 2500 & 3000 \\
\hline $\begin{array}{c}\text { Input External Excitation } \\
\text { Force, N }\end{array}$ & 4365 & 8154 & 9995 & 9995 & 9995 & 9350 & 9995 \\
\hline Frequency, Hz & 500 & 500 & 500 & 500 & 1000 & 1000 & 1000 \\
\hline$M P I, \mathrm{~W}$ & 294 & 550 & 647 & 674 & 1350 & 1262 & 1350 \\
\hline$E P U, \mathrm{~W}$ & 28 & 72.5 & 146 & 148 & 107 & 231 & 242 \\
\hline$P H, \%$ & 9.5 & 13 & 22 & 22 & 8 & 18 & 18 \\
\hline
\end{tabular}

Table 1: Data from measurements for circuit with resistance $1 \Omega$

In the Table 2 the data of experimentation with MEG for $0.5 \Omega$ resistance coil are presented. The $M P I, E P U, P H$ are calculated for different mechanical prestresses, input external excitation forces and frequencies.

\begin{tabular}{|c|c|c|c|c|c|c|c|c|c|}
\hline $\begin{array}{c}\text { Mechanical } \\
\text { Prestress, N }\end{array}$ & 1000 & 2000 & 2500 & 2000 & 2500 & 3000 & 3500 & 4000 & 4500 \\
\hline $\begin{array}{c}\text { Input External } \\
\text { Excitation Force, N }\end{array}$ & 6528 & 7905 & 9155 & 9995 & 8476 & 9995 & 9995 & 9995 & 9995 \\
\hline Frequency, Hz & 500 & 500 & 500 & 1000 & 1000 & 1000 & 1000 & 1000 & 1000 \\
\hline$M P I, \mathrm{~W}$ & 440 & 534 & 618 & 1350 & 1144 & 1350 & 1350 & 1350 & 1350 \\
\hline$E P U, \mathrm{~W}$ & 60 & 110 & 156 & 213 & 153 & 178 & 221 & 174 & 199 \\
\hline$P H, \%$ & 14 & 21 & 25 & 16 & 14 & 13 & 16 & 13 & 15 \\
\hline
\end{tabular}

Table 2: Data from measurements for circuit with resistance $0.5 \Omega$

Analysis of data of the Tables 1-2 shows that the maximum output power of the MEG is equal to 242 Watts, (See column 7 in Table 1).

From Fig. 6 (force Vs time curves for frequency $1000 \mathrm{~Hz}$ and for mechanical prestress $3000 \mathrm{~N}$ ) it can be found that there is some additional force (thin curve) that builds up in addition to the mechanical prestress applied (thick curve). The sum of these forces is called input external excitation force. It's can be noted from curves in Fig. 6 and Fig. 7 that the peaks of input external excitation force and the peaks of voltage with respect to time both rise in the same instants of time.

Analysis of experimental data has shown that Terfenol-D rod is sensitive to both mechanical prestress and frequency of external excitation, but more sensitive to frequency than mechanical prestress. For instance, increase in the mechanical prestress from $2000 \mathrm{~N}$ to 
$4500 \mathrm{~N}$ and keeping the frequency constant at $1000 \mathrm{~Hz}$, there is about $30 \%$ fluctuation of EPU values, (see Table 2). From the Table 1 and Table 2 it also follows that for mechanical prestress $2700 \mathrm{~N}$ and frequency $500 \mathrm{~Hz}$ the efficiency of the MEG was maximum for both 1 $\Omega$ and $0.5 \Omega$ resistance, even though the $E P U$ is lower than the highest $E P U$, (refer to Table 1, $6^{\text {th }}$ row and $8^{\text {th }}$ column). It may be right to assume that the MEG has not yet reached its highest potential limit for $1 \Omega$ resistance circuit.

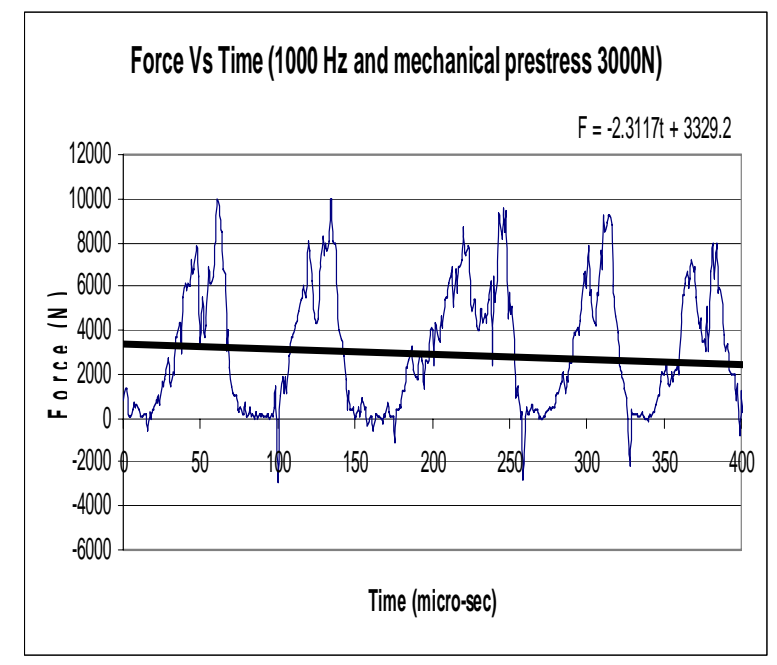

Figure 6. Force Vs Time for frequency $1000 \mathrm{~Hz}$, and mechanical prestress $3000 \mathrm{~N}$

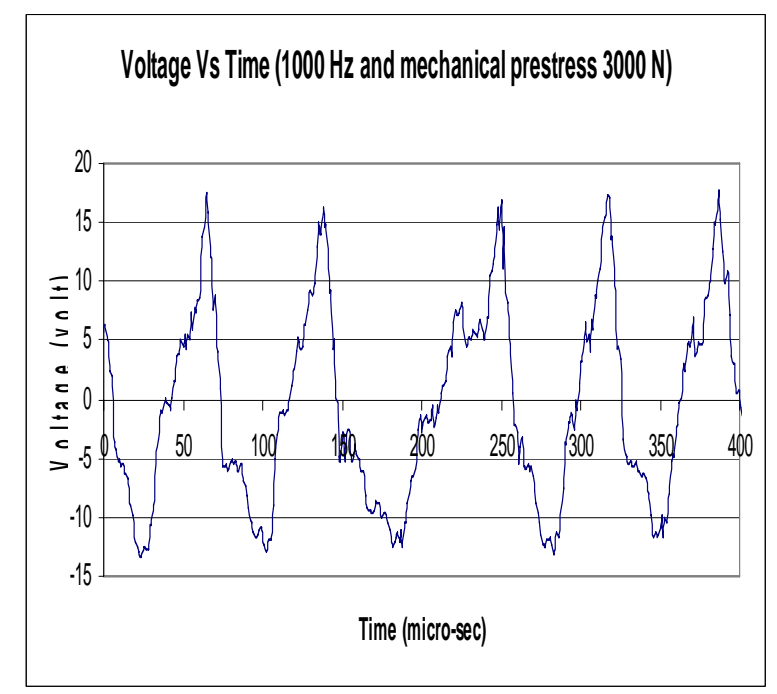

Figure 7. Voltage Vs Time for frequency $1000 \mathrm{~Hz}$, and mechanical prestress $3000 \mathrm{~N}$ 
The MEG voltage profile (See Fig. 7) is sinus wave. To have MEG to charge a battery, there would be a need to have a rectifier to produce continuous power supply. The rectifier need to be designed considering for both rising voltages and also when the voltage saturates, and hence not effecting the power supply to the device, (actuator or sensor). More detail results of experimental study of transduction processes in developed MEG can be found in [31].

\section{CONCLUSIONS}

The mathematical model has been developed for modeling of performance of magnetostrictive power harvesting devices. It comprises the constitutive equations of magnetoelastic behavior of magnetostrictive rod (active element of magnetostrictive transducer) and standard formulae of electromagnetism for induced voltage and current in the pick-up coil due to variation of magnetic field intensity. The algorithm is proposed to evaluate electrical power output of MEG for different external excitations. The algorithm was implemented in Matlab/Simulink. Its efficiency has been confirmed by simulation of performance of different MEG under the periodic excitations exerted by the "hosting" system.

The new MEG was built based on the utilization of Villary effect. Several experiments demonstrated its functionality to produce electric power for varying mechanical prestress and frequency of external excitations. Maximum electric power obtained was equal to $242 \mathrm{~W}$. For considered different mechanical prestresses and frequencies of external excitations the efficiency of the created MEG varies from $8 \%$ to $25 \%$, (see data in Table 1 and Table 2). The obtained results of experimental study of vibration-to-electric energy conversion using Terfenol-D demonstrate the potential of using giant magnetostrictive materials for power harvesting from vibration. These results can also be used for verification and validation of the mathematical models of magnetostrictive electric generators for predicting their performance and optimization of the generator design.

\section{ACKNOWLEDGMENTS}

This paper was written in the context of the MESEMA project, funded under the 6th Framework Programme of the European Community (Contract N AST3-CT-2003-502915).

\section{REFERENCES}

1. Handbook of Giant Magnetostrictive Materials, Edited by Göran Engdahl, Academic Press, San Diego, USA, 2000.

2. Claeysse F., Lhermet N., Le Letty R. and P.Bouchilloux, Actuators, transducers and motor based on giant magnetostrictive materials, Journal of Alloys and Compounds, 258, 1997, 61-73.

3. May C., Kuhnen K., Pagliarulo P., and H. Janocha, Magnetostrictive dynamic vibration absorber (DVA) for passive and active damping, Proc. Euronoise 2003, Naples, 2003, paper ID: 159. 
4. Sodano H.A., Inman D.J. and G. Park, A review of power harvesting from vibration using piezoelectric materials, The Shock and Vibration Digest, Vol. 36, No.3, May 2004, 197-205.

5. Glynne-Jones P., Tudor M.J., Beeby S.P. and N.M. White, An electromagnetic, vibrationpowered generator for intelligent sensor systems, Sensors and Actuators A 110 (2004) 344-349.

6. Lu F., Lee H.P., and S P Lim, Modeling and analysis of micro piezoelectric power generators for micro-electromechanical-systems applications, Smart Mater. Struct. 13 (2004) 57-63.

7. Meninger S, Mur-Miranda J O, Amirtharajah R., Chandrakasan A P and Lang J H 2001 Vibrationto-electric energy conversion IEEE Trans. Very Large Scale Integr. (VLSI) Syst. 9 64-76.

8. Roundy S. and P.K. Wright, A piezoelectric vibration based generator for wireless electronics, Smart Mater. Struct. 13 (2004) 1131-1142

9. Shenck N. S. and Paradiso J. A., Energy scavenging with shoe-mounted piezoelectrics, IEEE Micro ,2001, 21 30-41

10. Konak M.J., Powlesland I.G., van der Velden S.P., and S. C. Galea, A self-powered discrete time piezoelectric vibration damper," in Proc. SPIE_Int. Soc. Optical Eng., vol. 3241, 1997.

11. Amirtharajah R. and A. P. Chandrakasan, Self-powered signal processing using vibration-based power generation," IEEE J. Solid-State Circuits, vol. 33, May 1998.

12. Yamamoto, Y., Eda, H., Mori, T. and A. Rathore, Three-dimensional magnetostrictive vibration sensor: development, analysis, and applications, Alloys and Compounds, 258, 107-113, 1997.

13. Williams C.B., and R. B. Yates, Analysis of a micro-electric generator for microsystems", Sensors and Actuators, A 52, 1996, pp. 8-11.

14. James E.P., M.J. Tudor, S.P. Beeby, P. Glynne-Jones, J.N. Ross, N.M. White, A Wireless SelfPowered Micro-System for Condition Monitoring (Eurosensors XVI), Prague, 2002.

15. James E.P., M.J. Tudor, S.P. Beeby, N.R. Harris, P. Glynne-Jones, J.N. Ross, N.M. White, An investigation of self-powered systems for condition monitoring applications, Sensors and Actuators A 110 (2004) 171-176

16. El-hami M, Glynne-Jones P, White N M, Hill M, Beeby S, James E, Brown A D and Ross J N 2001 Design and fabrication of a new vibration-based electromechanical power generator Sensors Actuators A 92 335-42.

17. Glynne-Jones P., Beeby S. P., James E. P. and White N. M., The modelling of a piezoelectric vibration powered generator for microsystems Transducers 01/Eurosensors XV, June 2001

18. To T.K.H., W. Sun, and W. J. Li, "A wireless self-powered rotation sensing system using a vibration-based micro power transducer", IEEE/ASME M2VIP, Hong Kong, Aug 27-28, 2001.

19. Ching N.,H. Wong, W. Li, P. Leong, Z. Sar, U. Chongquing, in: A Laser Micromachined Vibrational to Electrical Power Transducer for Wireless Sensing Systems (Transducers 01), June 10-14, 2001, Munich, pp. 42-45.

20. Dapino M., F. Calkins, and A. Flatau, $\backslash \mathrm{On}$ indenti ${ }^{-}$cation and analysis of fundamental issues in Terfenol-D transducer modeling," Proceedings of SPIE Smart Structures and Materials Conf. 3392(23), 1998.

21. Dapino M.J., R.C. Smith and A.B. Flatau. Structural-magnetic strain model for magnetostrictive transducers. IEEE Trans. Magn., 36(3):545 \{556, 2000.

22. Dapino, M.J., R.C. Smith and A.B. Flatau, Structural-magnetic strain model for magnetostrictive transducers," IEEE Trans. Magn., 2000, 36(3): 545-556.

23. Janocha, H., Application potential of magnetic field driven new actuators, J. Sensors and Actuators, A91, 2001, pp.126-132.

24. Lundgren A., Tiberg H., Kvarnsjö L., Bergqvist A. and G. Engdahl, A magnetostrictive electric generator, IEEE Trans. Magn., Vol.29, No. 6, 1993, 3150-3152. 
25. Kvarnsjö L., On characterization, modeling and application of highly magnetostrictive materials, Doctoral Thesis, Royal Institute of Technology, Sweden, 1993.

26. Edgdahl, G., Design procedure for optimal use of giant magnetostrictive materials in magnetostrictive actuator applications, in Proc. Of 8th International Conference on New Actuators, ACTUATOR2002, 10-12 June 2002, Bremen, Germany, 2002, pp. 554-557.

27. Cedell T., Magnetostrictive materials and selective application, Doctoral Thesis, Lund University, Sweden, 1996.

28. Jiles D.C., and Devine M.K., The law of approach as a means of modelling the magnetomechanical effect, J. Magn. Magn. Mater., 140-144, 1995, 1881-1882.

29. Li L., and Jiles D.C., Modeling of the magnetomechanical effect: Application of the Rayleigh law to the stress domain, Journal of Applied Physics, Vol.93, No 10, 2003, 8480-8482.

30. Benbouzid M.E.H., Kvarnsjö L. and G. Engdahl, Dynamic modelling of giant magnetostriction in Terfenol-D rod by the Finite Element Method, IEEE Trans. Magnetics, Vol.31, No 3, 1995, 1821 1824.

31. Berbyuk, V., Sodhani, J. and J. Möller, "Experimental study of power harvesting from Vibration using giant magnetostrictive materials", In Proc. $1^{\text {st }}$ International Conference on Experiments, Processes, System Modelling, Simulation and Optimization, Athens, Greece, July 6-9, 2005. 